\title{
Radiation induced by relativistic electron showers in the X-ray spectrum of Active Galactic Nuclei
}

\author{
A. Antonicci ${ }^{1}$ and A. I. Gómez de Castro ${ }^{2}$ \\ ${ }^{1}$ Dipartimento di Fisica G. Occhialini, Universita' degli Studi di Milano-Bicocca, Pza. della Scienza 3, 20126 Milano, Italy \\ 2 Instituto de Astronomía y Geodesia (CSIC-UCM), Fac. de CC Matemáticas, Universidad Complutense, \\ 28040 Madrid, Spain \\ e-mail: aig@mat.ucm.es
}

Received 2 August 2004 / Accepted 8 November 2004

\begin{abstract}
The iron $\mathrm{K} \alpha$ emitted from accreting black holes is thought to be produced by the reprocessing of hard X-ray radiation illuminating the disk. Mechanisms which could produce this hard X-ray radiation are magnetic reconnection in the disk corona or shocks. Both phenomena produce high energy particles whose contribution is usually ignored. In this work, we analyze how the transfer of mechanical energy from relativistic electrons to the circumnuclear gas (accretion disk, BLR) contributes to the $\mathrm{X}$-ray continuum and the iron $\mathrm{K} \alpha$ emission. It is shown that for gas columns comparable to the Thomson depth, the iron K $\alpha$ yield is comparable to that observed provided that the electron energy is above $\sim 600 \mathrm{keV}$ and that the total kinetic luminosity of the beam is around $\log L_{\mathrm{KIN}}=46.6-47.7$; this luminosity is comparable to that observed in radio-loud AGNs. The photon index of the X-ray continuum ( $8 \mathrm{keV}-20 \mathrm{keV})$ generated in such an electron shower is $1 \leq \Gamma \leq 2$. $\Gamma$ and the continuum strength are strongly model-dependent; they are dependent on both the relative orientation between the electron beam and the observer and the radius of the electron beam compared with the characteristic radius of the absorbing medium. The relevance of particle energy transport compared to photon energy transport in the AGN environment is outlined.
\end{abstract}

Key words. X-rays: general - X-rays: galaxies - galaxies: active

\section{Introduction}

Pound et al. (1990) pointed out that some of the most conspicuous features of the X-ray spectrum of AGN, could be produced by the reprocessing of hard X-ray radiation in the accretion disk. Even now, the nature of the hard X-ray source is still under debate. Two models have been proposed: the "lamppost" and the "hot corona" (Collin et al. 2003). The lamppost model assumes that the hard X-ray source is located on the disk axis, some few tens of the gravitational radius $\left(G M / c^{2}\right)$ above the disk. The origin of this source remains unknown; it has been suggested that it could associated with refocusing shocks at the base of magnetized disk winds (Pelletier \& Pudritz 1992). The hot corona model postulates a flaring hot corona over the magnetized accretion disk. Balbus \& Hawley (1991) showed that weak magnetic fields provide an efficient mechanism for angular momentum transport in accretion disks: weak fields provide a tension force that allows two orbiting fluid elements to exchange angular momentum on larger scales than the hydrodynamical viscosity scales. The Magneto Rotational Instability (MRI) acts like a dynamo, amplifying the field which is lost due to buoyancy, leading to a magnetized corona. Flares associated with reconnection events would naturally be produced in both models and, as a consequence, relativistic beams of elec- trons are generated at the same time as hard X-ray photons. The possible contribution from these beams to the $\mathrm{X}$-ray spectrum has often been ignored. The main reason is well summarized in a recent work by Ballantyne \& Fabian (2003) which is focused on the production of $\mathrm{Fe}-\mathrm{K} \alpha$ photons: electrons (and protons) need to be $10^{2}-10^{4}$ times more energetic than photons to produce the same radiative output as hard X-ray photons. In the case of the $\mathrm{Fe}-\mathrm{K} \alpha$ line, this is because the cross-section for photoionization in the $1-100 \mathrm{keV}$ range is several orders of magnitude larger than the electron impact cross section. However, this argument is somewhat misleading since, e.g., in solar flares the electrons reach energies in the $\mathrm{MeV}$ range during the impulsive phase; the onset of plasma microinstabilities accelerates the electrons to relativistic velocities during this phase (see e.g. Heyvaerts et al. 1977). In this work, we evaluate the output $\mathrm{X}$-ray radiation from the propagation of relativistic electrons (from the several hundred $\mathrm{keV}$ of radio-jets to some $\mathrm{MeV}$ ) in the dense matter surrounding the AGNs. Our objective is to determine the physical conditions under which electron impact produces an X-ray flux comparable to that generated by photoionization. Notice that these physical conditions may apply only to a fraction of the AGN. 
The propagation of a high-energy particle generates a cascade of secondary particles that represents an effective degradation of energy or shower which can be evaluated using a Monte Carlo code due to its random nature. We have adapted for the study of this problem PENELOPE: a Monte Carlo code initially designed to study the propagation of electron-photon showers with energy from $1 \mathrm{keV}$ to several hundreds $\mathrm{MeV}$ in arbitrary materials (see Baró et al. 1994). In Sect. 2, an overall description of the code is given. The numerical experiments run to simulate the propagation of electron beams in the AGN environment are described in Sect. 3. In Sect. 4 the results from the numerical simulations are compared to observations. A brief discussion on the relevance of particle energy transport within the AGN paradigm is included in Sect. 5. The major conclusions of this work are summarized in Sect. 6 .

\section{A numerical code to study the propagation of relativistic beams of electrons in dense gas}

The propagation of a high-energy particle generates a cascade of secondary particles; after each interaction, the energy of the primary (or secondary) particle is reduced so that the evolution represents an effective degradation of the energy in the shower. With time, the initial energy is progressively deposited into the medium, while that remaining is shared by an increasingly larger number of particles. The evolution of an electronphoton shower is of a random nature, so that this is a process particularly amenable to Monte Carlo simulation. The simulation of photon transport is straightforward since the mean number of events in each history is fairly small. Indeed, high energy photons are effectively absorbed after a single photoelectric or pair-production interaction or after a few Compton interactions. The simulation of electron and positron transport is much more difficult since the average energy loss by an electron in a single interaction is very small (around a few tens of eV). As a consequence, high-energy electrons suffer a large number of interactions before being effectively absorbed in the medium. In practice, detailed simulation is feasible only when the average number of collisions in the path is not too large. For highenergy electrons and positrons, most of the Monte Carlo codes currently available rely on multiple scattering theories which allow the simulation of the global effect of a large number of events in a track segment of a given length (or step). These simulation procedures are refered to as "condensed" Monte Carlo methods since the global effect of a large number of events is condensed in a single step. In this work, we use the program developed by Antonicci (2003) to simulate the propagation of fast electrons in plasmas. This program runs a subroutine package named PENELOPE which is a Monte Carlo code ${ }^{1}$ initially designed to study the propagation of electron-photon showers,

\footnotetext{
1 The Monte Carlo algorithm implemented in PENELOPE incorporates a "mixed" scattering model for the simulation of electron and positron transport. Hard interactions, with scattering angle and/or energy loss greater than preselected cutoff values are simulated in detail, by using simple analytical differential cross sections for the different interaction mechanisms and exact sampling methods. Soft interactions, with scattering angle or energy loss less than the corresponding cutoffs, are described by means of a multiple scattering
}

with energy from $100 \mathrm{keV}$ to several hundred $\mathrm{MeV}$, in arbitrary materials (see Baró et al. 1994, and references therein). The cross sections for hard elastic scattering, hard inelastic collisions, hard bremsstrahlung emission, soft artificial events and positron annihilation are taken into account to calculate the interactions of the electrons with matter. The following cross sections have been taken into account for the interaction of the secondary photons with the cloud: coherent (Rayleigh) scattering, incoherent (Compton) scattering, photoelectric absorption of photons and electron-positron pair production. We have set a minimum energy threshold of $1 \mathrm{keV}$ for the electrons since electrons with lower energy are not able to induce significant radiative effects in the $\mathrm{X}$-ray range in the gas. The program is described in full detail in Antonicci (2004). It has also been adapted to simulate fast electron propagation in solid matter within the context of laser-matter interaction. This has allowed validating the collisional part of the code by comparing the computational results with the experimental results from LULI (Laboratoire pour l'utilisation de lasers intenses) and from the Livermore laboratory ${ }^{2}$.

\section{The output spectrum}

The set-up for the numerical experiment is summarized in Fig. 1 and described in detail below. The collimated beam of relativistic electrons impinges on a spherical cloud. The output spectrum is calculated for three orientations:

1. normal to electron beam (scattered spectrum);

2. along the beam direction from the rear of the cloudlet (transmitted spectrum);

3. along the beam direction from the hot spot in the cloudlet (reflected spectrum). This would also represent the spectrum back-illuminating the source of electrons.

Notice that there are two main regions in AGNs able to produce X-ray radiation by this mechanism: the accretion disk surface and the dense cloudlets in the BLR. In the first case, the electron beam impacts on the disk surface heating it up and the observed X-ray spectrum would be like the reflected spectrum. In the second case, the electron beams impinge on small cloudlets: the relative orientation between the cloudlet, the electron beam and the observer will favor the observation of the scattered/transmitted spectrum. The final observed spectrum will be a mixture of these two orientations.

approach. The recently developed PENELOPE 2001 Monte Carlo code is user-friendly and incorporates photon cross-section data from the EPDL97 which includes new libraries for the low-energy photon cross-sections, such as XCOM and EPDL97. The code is available at the web site of the International Atomic Energy Agency (URL: www.iaea.org/inis/ws/d3/r2123.html)

2 The code is, in addition, widely used in the medical community and, for instance, Sung-Joon et al. (2004) have recently verified it for clinical dosimetry of high-energy $(10 \mathrm{keV}-150 \mathrm{keV})$ electron and photon beams. 


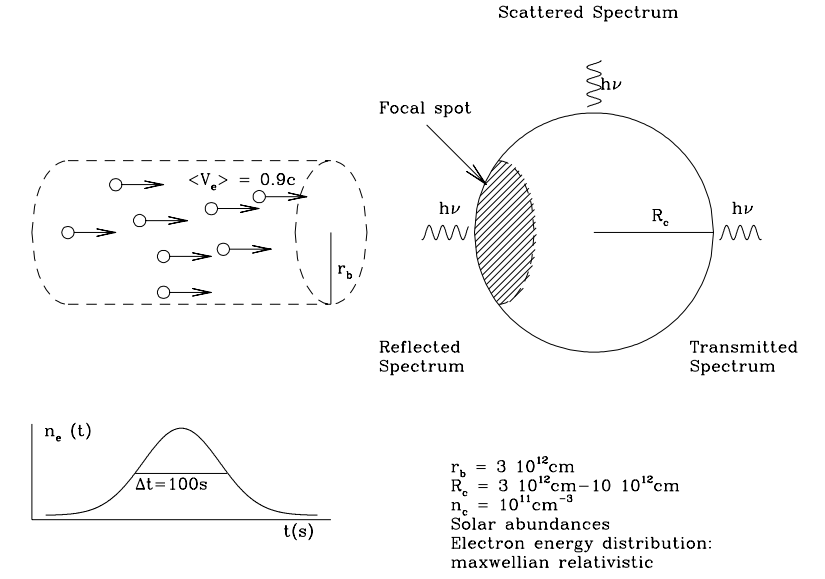

Fig. 1. Summary of the numerical experiment set-up. The cylindrical beam impinges on a spherical cloud with radius, $R_{\mathrm{c}}$. The electrons interact with the cold cloud atoms producing radiation. If the electrons are not very energetic, most of their kinetic energy is released at the front of the cloud producing a "focal hot spot". As the energy increases, the electrons penetrate further in the cloud and the radiation generated in shower is emitted in all directions. The output X-ray spectrum has been studied for three orientations of the line of sight: normal to the electron beam (scattered spectrum), along the beam direction from the rear of the cloudlet (transmitted spectrum) and along the beam direction from the hot spot in the cloudlet (reflected spectrum). The electron beam is not uniform: the electron density distribution, $n_{\mathrm{e}}$, depends on the radial distance to the beam axis, $r$, as $n_{\mathrm{e}}=n_{0} \exp \left(-\left(r / r_{\mathrm{b}}\right)^{2}\right)$, where $r_{\mathrm{b}}$ parametrizes the characteristic width of the beam. The electrons are injected in a single pulse with characteristic time $100 \mathrm{~s}$ (see the bottom inset).

The main assumptions built-in the simulation are:

1. The electron energy distribution is Maxwellian relativistic ${ }^{3}$. The electron beam is characterized by its mean kinetic energy. Kinetic energies between $30 \mathrm{keV}$ and $2.3 \mathrm{MeV}$ have been considered (e.g. well above the $7.1 \mathrm{keV}$ threshold for the production of $\mathrm{K} \alpha$ emission by electron collision). Above $30 \mathrm{keV}$, the cross-section for K-shell ionization is significantly larger for collisional processes than for photoionization (Verner \& Yakovlev 1995; Hombourger 1998). The upper energy limit, $2.3 \mathrm{MeV}$, corresponds to a highly relativistic beam with mean velocity $0.983 \mathrm{c}$.

The electron energy distribution has been assumed to be Gaussian to control the response of the medium to a given electron energy. A detailed analysis of the response to power law electron energy distribution is the subject of a further article since the response of the medium to a power-law spectrum depends strongly on the high energy

${ }^{3}$ The distribution function of the number of electrons $\left(n_{\mathrm{e}}\right)$ per kinetic energy $(K)$ is given by:

$\frac{\mathrm{d} n_{\mathrm{e}}}{\mathrm{d} K}=\gamma\left(\gamma^{2}-1\right)^{1 / 2} \mathrm{e}^{-K / k_{\mathrm{B}} T_{\mathrm{e}}}$

where $\gamma=\left(1-(v / c)^{2}\right)^{-1 / 2}, K=0.511 \mathrm{MeV}(\gamma-1), k_{\mathrm{B}}$ is the Boltzmann constant and $T_{\mathrm{e}}$ is the electron temperature. The mean kinetic energy of the electron beam is $\sim 3 k_{\mathrm{B}} T_{\mathrm{e}}$ since the distribution has a broad tail toward high energies. The mean kinetic energy is used to define the beam energy. threshold which is a very uncertain parameter (for instance, for a power law $\mathrm{d} N / \mathrm{d} E=E^{-2.6}$ with an upper energy threshold of $2.2 \mathrm{MeV}$, the average energy of the beam is $1.2 \mathrm{keV}$ and therefore, most of the electrons are absorbed without producing significant $\mathrm{X}$-ray radiation).

2. The beam luminosity is controlled by the injection time, e.g. it is defined as the total number of fast electrons weighted with the energy distribution and divided by the injection time. The injection is modeled with a Gaussian temporal distribution of the beam with Full Width Half Maximum (FWHM) $100 \mathrm{~s}$. As our code is a time dependent Monte Carlo code, it is possible to see the time evolution of the emitted spectrum and then study how it saturates. The history of the injected electrons is divided in time steps of $\sim 1 \mathrm{~s}$ and the evolution of their propagation is studied during a time interval of $300 \mathrm{~s}$, e.g., the time necessary for most of the electrons (and photons) to be absorbed in the medium or to escape the cloud. Photon and electron counting is carried out during this time lapse.

3. The beam impinges on a spherical cloudlet with uniform density equal to $n_{\mathrm{c}}=10^{11} \mathrm{~cm}^{-3}$ which is the characteristic density of the gas in the BLR. Several cloud radii, $R_{\mathrm{c}}$, have been used to test the variation of the spectrum with the gas column in the transition regime between Thomson thin and thick gas columns ${ }^{4}$. The fiducial radius, $r_{0}$, has been selected so that,

$2 r_{0} * n_{\mathrm{c}}=\frac{1}{1.2 \sigma_{T}}$

or, $r_{0}=6.3 \times 10^{12} \mathrm{~cm}$. Models from $R_{\mathrm{c}}=3 \times 10^{12} \mathrm{~cm}$ to $R_{\mathrm{c}}=10^{13} \mathrm{~cm}$ have been run.

4. The cloudlet is assumed to be cold and with solar-like abundances. Only the most abundant elements from hydrogen to sulfur are included (hydrogen, helium, oxygen, carbon, neon, nitrogen, magnesium, silicon, iron and sulfur).

5. The radial distribution of the incident electrons is assumed to depend on the distance to the beam axis. This distribution beam is parametrized with a Gaussian function so the number of electrons, $n_{\mathrm{e}}$, impinging on the the cloud at a radius, $r$, from the beam axis is $n_{\mathrm{e}}=n_{0} \exp \left(-\left(r / r_{\mathrm{b}}\right)^{2}\right)$ where $n_{0}$ is the number of electrons on the beam axis and $r_{\mathrm{b}}$ is a characteristic radius, a measure of the width of the beam. All the particles up to a radius twice $r_{\mathrm{b}}$ have been considered. $r_{\mathrm{b}}$ has been set equal to $3 \times 10^{12} \mathrm{~cm}$ in most of this work (see Sect. 3.3 for a test on the effect of $r_{\mathrm{b}}$ in the output spectrum). This value roughly corresponds to a tenth of the inner disk radius ${ }^{5}$ for a fiducial $M_{\mathrm{bh}}=10^{8} M_{\odot}$ black hole.

We have run tests increasing the number of test particles to check the statistics and the spectrum converge

\footnotetext{
${ }^{4}$ Assuming a $10 \%$ abundance of helium by number.

${ }^{5}$ Recent observations suggest that the inner radius of the disk is around $2 R_{\mathrm{S}}$, where $R_{\mathrm{S}}$ is the Schwarzschild radius:

$R_{\mathrm{S}}=\frac{2 G M_{\mathrm{bh}}}{c^{2}}=2.95 \times 10^{13} \mathrm{~cm}\left(\frac{M_{\mathrm{bh}}}{10^{8} M_{\odot}}\right)$

and $M_{\mathrm{bh}}$ is the mass of the black hole.
} 


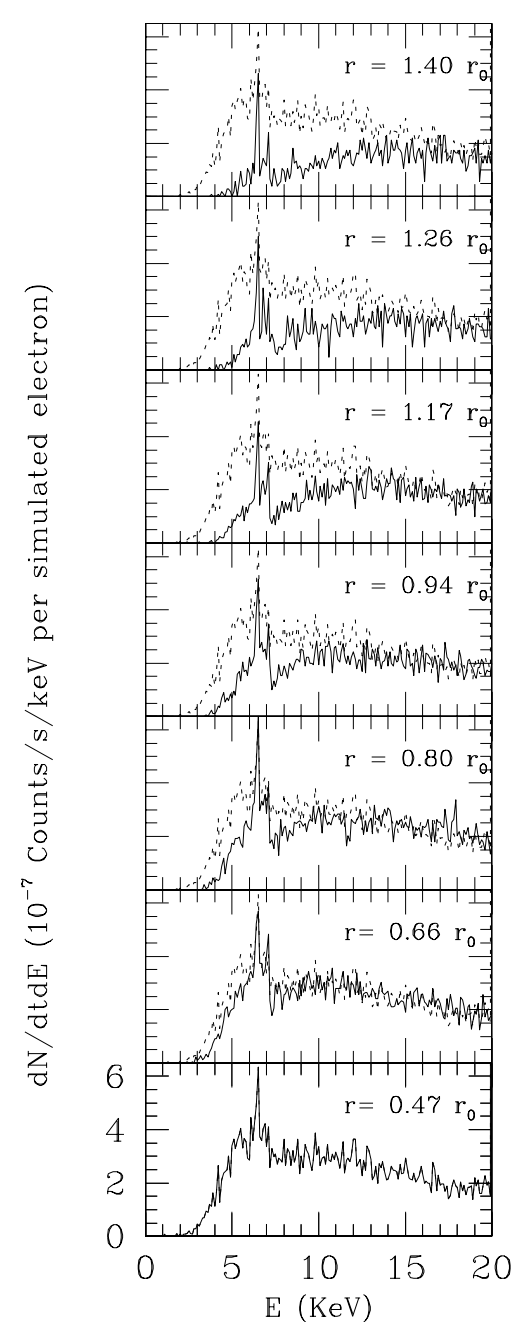

Fig. 2. Output transmitted X-ray spectrum from a cloud heated by a beam of relativistic electrons $(0.9 \mathrm{c}) . r_{0}$ is the fiducial cloud radius: $6.3 \times 10^{12} \mathrm{~cm}$ which traces the transition from Thomson thin to Thomson thick gas columns (see text). The spectrum for $r=0.47 r_{0}$ has been over-plotted (dashed line) in all the panels to illustrate how the increasing gas column modifies the continuum energy distribution.

rapidly: 20 million test particles are enough to get meaningful results with a counting bin of $10 \mathrm{eV}$. Also we have carried out an explicit calculation of the $\mathrm{Fe}-\mathrm{K} \alpha$ yield.

\subsection{The output spectrum and the cloudlet thickness}

We have run a grid of simulations with different cloudlet radius from $3 \times 10^{12} \mathrm{~cm}$ to $10^{13} \mathrm{~cm}$ and a relativistic $\left(\left\langle V_{\mathrm{e}}\right\rangle=\right.$ $0.9 \mathrm{c}$ ) beam (see Fig. 2). The X-ray continuum is non-thermal bremsstrahlung radiation from the interaction of the relativistic electrons with the cloud (Bethe \& Heitler 1934). This radiation is partially absorbed by the cloud. The effect is clearly seen in Fig. 2: low energy photons are absorbed and the low energy cut-off moves to higher energies as the gas column increases, from $6 \times 10^{23} \mathrm{~cm}^{-2}$ at the bottom to $1.8 \times 10^{24} \mathrm{~cm}^{-2}$ at the
Table 1. Characteristics of the beam absorption.

\begin{tabular}{lccc}
\hline \hline $\begin{array}{l}R_{\mathrm{c}} \\
\left(10^{12} \mathrm{~cm}\right)\end{array}$ & $\left\langle N_{\mathrm{H}}\right\rangle^{a}$ & $\begin{array}{c}R_{\mathrm{abs}}^{b} \\
\left(N_{T}\right)\end{array}$ & $\frac{L_{\mathrm{t}}^{c}}{L_{\mathrm{s}}}$ \\
\hline 3 & 0.48 & 0.785 & 2.92 \\
4 & 0.64 & 0.916 & 2.54 \\
5 & 0.80 & 0.996 & 2.32 \\
6 & 0.96 & 0.998 & 2.15 \\
7 & 1.12 & 0.998 & 1.98 \\
8 & 1.28 & 0.998 & 1.97 \\
9 & 1.44 & 0.998 & 1.83 \\
10 & 1.60 & 0.998 & 1.73 \\
\hline
\end{tabular}

a Gas column at the center of the beam in units of $N_{T}$ $\left(1.25 \times 10^{24} \mathrm{~cm}^{-2}\right)$.

${ }^{b}$ Percent of the incident electrons that are absorbed by the cloud.

${ }^{c}$ Rate between the transmitted, $L_{\mathrm{t}}$, and the scattered, $L_{\mathrm{s}}, \mathrm{X}$-ray radiation.

top. The iron $\mathrm{K} \alpha$ feature is very prominent ${ }^{6}$ and has a maximum strength for a radius of $6 \times 10^{12} \mathrm{~cm}: 1.8 \times 10^{-7}$ counts $/ \mathrm{s}$ per incident electron. This radius corresponds to the transition from Thomson thin to Thomson thick columns. Also the iron absorption edge at $7.1 \mathrm{keV}$ is reproduced by the simulations. The fraction of the incident energy directly radiated in the $1-20 \mathrm{keV}$ range is about $10^{-5}$ times the mechanical energy injected in the cloud and it is not radiated in an isotropic manner (see Table 1); the transmitted spectrum is stronger than the scattered one when the gas column is small, but as the gas column increases the radiation becomes more isotropic because of the straggling of the energy loss in the shower process (see Table 1). Most of the electron energy is damped into gas heating.

\subsection{The output spectrum and the kinetic energy of the electron beam}

We have run several experiments changing the kinetic energy of the electron beam impinging on the cloud from $30 \mathrm{keV}$ to $2297 \mathrm{keV}$ (see Figs. 3 and 4). The main conclusions are:

1. For small energies $(E<100 \mathrm{keV}$ per electron) the electrons are rapidly absorbed so significant $\mathrm{X}$-ray radiation is only observed from the hot spot at the front of the cloud. This is bremsstrahlung radiation from the interaction of the relativistic electrons with the cold gas. The reflected photon counts have a power-law dependence: $\mathrm{d} N(E) / \mathrm{d} E \propto$ $E^{-1.00 \pm 0.05}$ for $E=30 \mathrm{keV}$ (see Fig. 3). The $\mathrm{K} \alpha$ emission is negligible for $E<100 \mathrm{keV}$, confirming the small penetration depth of the beam.

2. For high energy beams $(E \simeq 2.2 \mathrm{MeV}$ per electron) the electrons are able to reach the back of the cloud $(99.7 \%$ of their kinetic energy is absorbed in the path). In this process,

${ }^{6}$ Although nickel, copper, titanium and zinc have strong $\mathrm{K} \alpha$ and $\mathrm{K} \beta$ features in the $6-10 \mathrm{keV}$ range, they are not included in the calculation (see Sect. 3). 


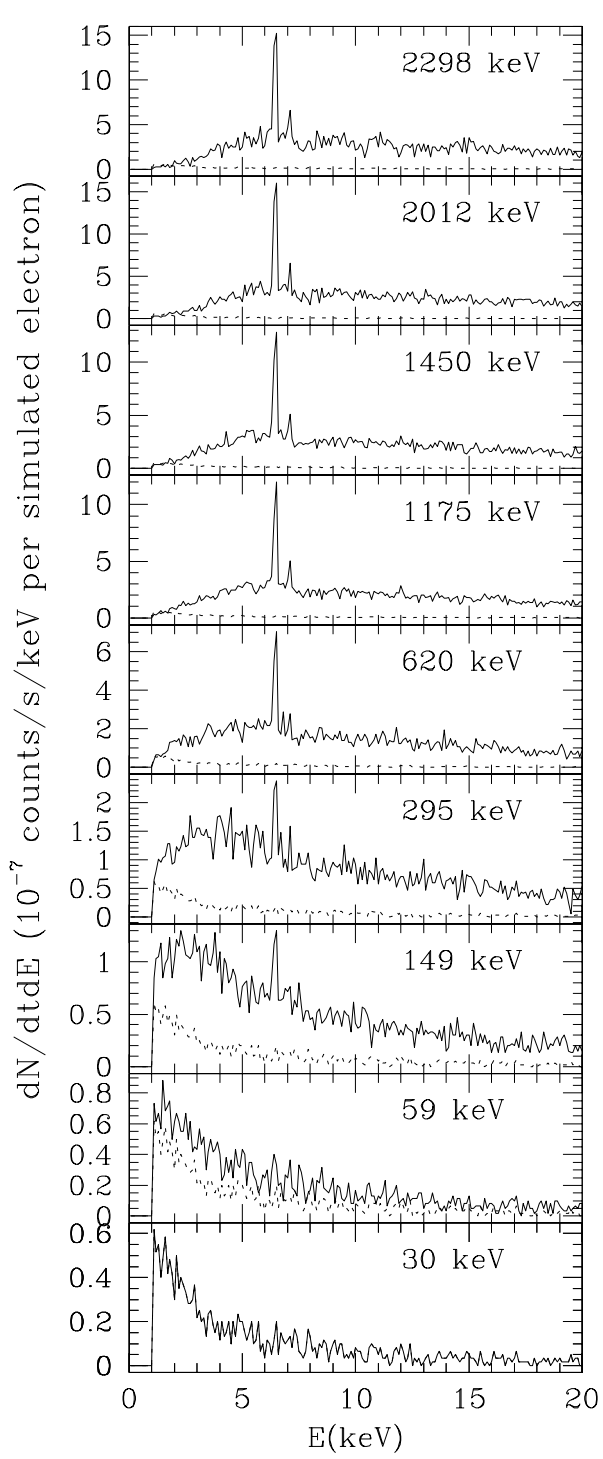

Fig. 3. Reflected X-ray spectrum from a spherical cloud, with radius $6 \times 10^{12} \mathrm{~cm}$, heated by beams of relativistic electrons with energies from 30 to $2.3 \mathrm{MeV}$ (from bottom to top).The spectrum with $E=30 \mathrm{keV}$ has been over-plotted (dashed line) in all the panels to illustrate how the increasing penetration of the electron beam on the cloud modifies the continuum energy distribution.

a tiny fraction of their kinetic energy is damped into high energy X-ray photons. Also significant $\mathrm{K} \alpha$ emission is produced and the iron edge at $7.1 \mathrm{keV}$ is clearly observed in the transmitted spectrum.

3. An intermediate trend is observed for intermediate energies: transmitted and reflected spectra have similar strengths. The observed hard X-ray energy distribution depends on the penetration of the beam within the cloud, e.g. on the absorption.

In all cases, the energy radiated in the $1 \mathrm{keV}-20 \mathrm{keV}$ range represents a tiny fraction of the kinetic energy of the beam ${ }^{7}$ (see Table 2 and Fig. 5). The penetration of the electrons into the cloud increases with the beam energy and, as a consequence,

\footnotetext{
${ }^{7}$ Only for highly relativistic electrons, with $\gamma=\left(1-(v / c)^{2}\right)^{-1 / 2} \gg$ 1 , energy loss by radiation predominates over collisional energy loss.
}

the $\mathrm{X}$-ray continuum radiation becomes more anisotropic; at $2.4 \mathrm{MeV}$ roughly a $70 \%$ of the X-ray continuum comes from the rear of the cloud (the transmitted spectrum). This is not the case of the Fe-K $\alpha$ line radiation (see Fig. 5) which is rather isotropic due to the progress of the "shower".

\subsection{The output spectrum and the beam radius}

If the electrons are generated during "flares" associated with disk coronal loops we should expect that the radius of the electron beams are significantly smaller than stated above. It is difficult to estimate this radius since the physical conditions of the disk (and especially the properties of the disk magnetic field and the circumnuclear field) are not known. The radius of a typical magnetic loop in a solar flare is $\sim 10^{-6}$ the length of the loop; as a first approach we will adopt this value.

The length of the loop is expected to be similar to the scaleheight of the disk, $H$,

$$
H(R) \simeq\left(\frac{2 k_{\mathrm{B}} T(R)}{G M_{\mathrm{bh}} \mu m_{\mathrm{H}}}\right)^{1 / 2} R^{3 / 2}
$$

where $\mu$ is the mean molecular weight, $m_{\mathrm{H}}$ is the mass of the hydrogen atom, $R$ is the distance to the disk axis and $T$ is the disk temperature at radius $R$,

$$
T(R)=6.3 \times 10^{5} \mathrm{~K}\left(\frac{\dot{M}}{\dot{M}_{E}}\right)^{1 / 4} M_{\mathrm{bh}, 8}^{-1 / 4}\left(\frac{R}{R_{\mathrm{S}}}\right)^{-3 / 4}
$$

which is given by the standard thin accretion disk formula (Peterson 1997) where $\frac{\dot{M}}{\dot{M}_{E}}$ is the ratio between the accretion rate and the Eddington rate and $M_{\mathrm{bh}, 8}$ is the black hole mass in units of $10^{8}$ solar masses. Therefore, if the loops are generated at a radial distance from the central black hole of $9 R_{\mathrm{S}}$ (Collin et al. 2003), the beam radius, $r_{\mathrm{b}}$, is expected to be,

$r_{\mathrm{b}} \simeq 10^{-6} H\left(9 R_{\mathrm{S}}\right)=1.14 \times 10^{5} \mathrm{~cm}\left(\frac{\dot{M}}{\dot{M}_{E}}\right)^{1 / 8} M_{\mathrm{bh}, 8}^{-1 / 8}$.

We have run a simulation with this beam radius for an energy of $2012 \mathrm{keV}$. As expected, the Fe-K $\alpha$ flux is not affected significantly but the continuum drops severely and it is only significant in the transmitted spectrum (see Fig. 6).

\section{Comparison with the observations}

As shown above, the combination of the X-ray radiation produced in the electron energy degradation shower and the cloud absorption produces a rather steep X-ray spectrum and strong iron emission. In the following, we make some estimates of the possible relevance of this mechanism in AGNs.

\subsection{The Fe-Ko flux}

There are two possible sources of Fe-K $\alpha$ flux in the AGNs environment associated with electron beam impact: the BLR clouds and the accretion disk. In the first case, the observed X-ray spectrum is dominated by the scattered component since we do not expect that the observer is aligned with the electron beam. In the second case, the spectrum is dominated by the 

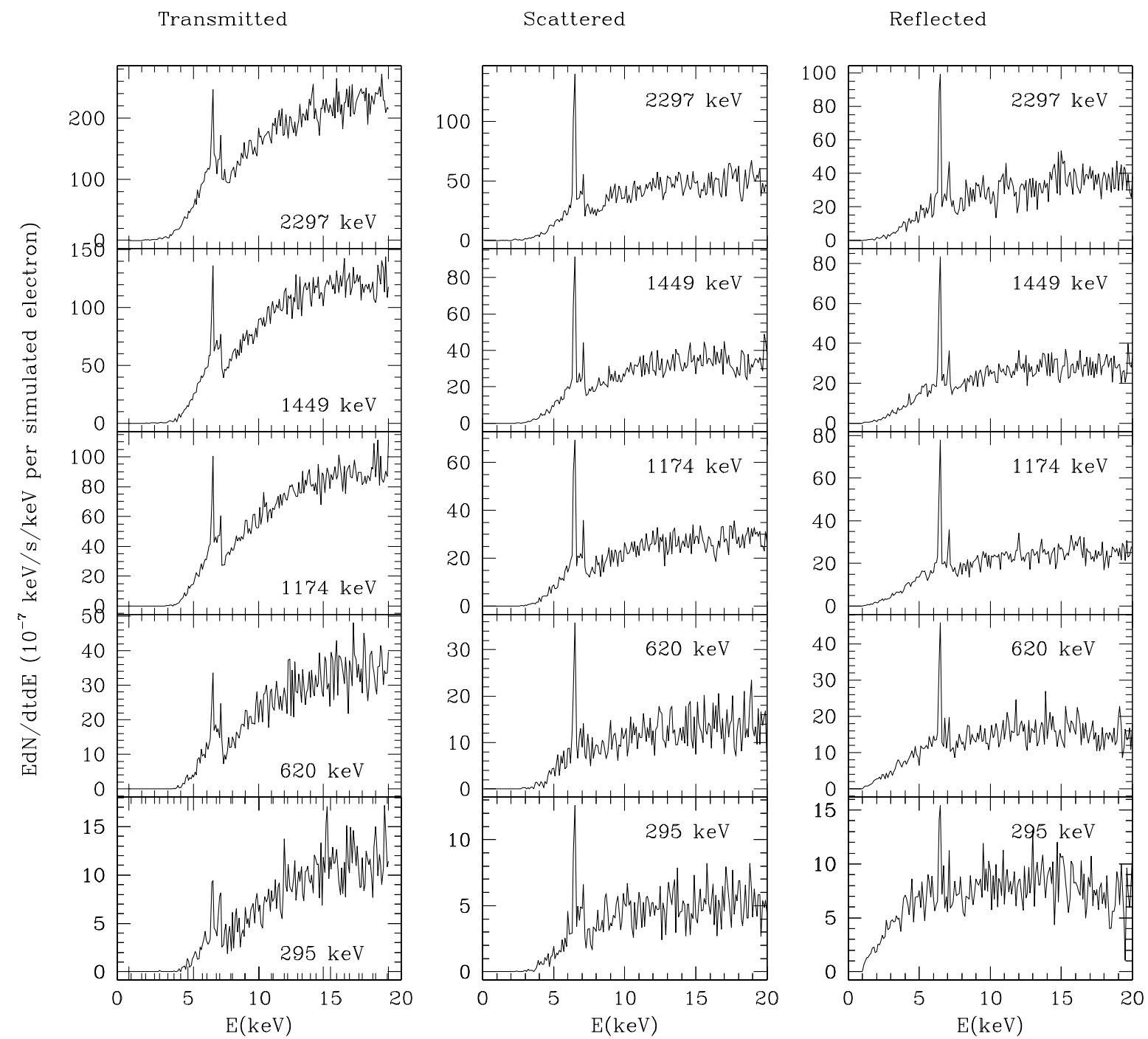

Fig. 4. Output transmitted, scattered and reflected X-ray spectrum from a spherical cloud, with radius $6 \times 10^{12} \mathrm{~cm}$, heated by beams of relativistic electrons with energies from $295 \mathrm{keV}$ to $2.3 \mathrm{MeV}$ (from bottom to top).

Table 2. X-ray radiation and electron energy.

\begin{tabular}{lccccc}
\hline \hline $\begin{array}{l}E \\
(\mathrm{keV})\end{array}$ & $\frac{L_{\mathrm{X}}}{L_{\text {beam }}}$ & $\frac{L_{\mathrm{t}}}{L_{\mathrm{X}}}$ & $\frac{L_{\mathrm{S}}}{L_{\mathrm{X}}}$ & $\frac{L_{\mathrm{b}}}{L_{\mathrm{X}}}$ & $\begin{array}{c}\epsilon_{\mathrm{K} \alpha}^{a}(E) \\
(\text { counts/s/str })\end{array}$ \\
\hline 2298 & $2.26 \mathrm{e}-3$ & 0.71 & 0.17 & 0.13 & $5.9 \mathrm{e}-8$ \\
2012 & $1.96 \mathrm{e}-3$ & 0.68 & 0.17 & 0.14 & $5.23 \mathrm{e}-8$ \\
1450 & $1.34 \mathrm{e}-3$ & 0.62 & 0.20 & 0.18 & $3.78 \mathrm{e}-8$ \\
1175 & $1.03 \mathrm{e}-3$ & 0.58 & 0.21 & 0.20 & $3.12 \mathrm{e}-8$ \\
620 & $1.32 \mathrm{e}-4$ & 0.48 & 0.22 & 0.30 & $1.41 \mathrm{e}-8$ \\
\hline
\end{tabular}

${ }^{a}$ Number Fe-K $\alpha$ photons per second and stereo-radian and simulated electron.

reflected component. However, for high enough energies, the $\mathrm{Fe}-\mathrm{K} \alpha$ yield is mostly independent of the orientation (see bottom panel in Fig. 5).
The line flux detected at a distance $d$ from the AGN depends on the $\mathrm{Fe}-\mathrm{K} \alpha$ yield, $\left\langle\epsilon_{\mathrm{K} \alpha}(E)\right\rangle$, as,

$F_{\mathrm{K} \alpha}\left(\frac{\text { counts }}{\mathrm{s} \mathrm{\textrm {cm } ^ { 2 }}}\right)=1.04 \times 10^{-49}\left\langle\epsilon_{\mathrm{K} \alpha}(E)\right\rangle N_{\mathrm{e}}\left(\frac{d}{1 \mathrm{Mpc}}\right)^{-2}$

where,

$\left\langle\epsilon_{\mathrm{K} \alpha}(E)\right\rangle=\frac{L_{\mathrm{K} \alpha, t}+L_{\mathrm{K} \alpha, b}+L_{K \alpha, s}}{4 \pi}$

so, $\left\langle\epsilon_{\mathrm{K} \alpha}(E)\right\rangle \simeq 1 \times 10^{-7}$ counts/strd/s for the energy ranges considered (see Fig. 7) and,

$F_{\mathrm{K} \alpha}\left(\frac{\text { counts }}{\mathrm{s} \mathrm{cm}^{2}}\right)=1.04 \times 10^{-56} N_{\mathrm{e}}\left(\frac{d}{1 \mathrm{Mpc}}\right)^{-2}$.

Notice also that this expression represents an upper limit to the true electron flux required to produce the observed $\mathrm{Fe}-\mathrm{K} \alpha$ flux since a fraction of the electrons will leave the cloudlet with enough energy to produce more $\mathrm{Fe}-\mathrm{K} \alpha$ photons if further cloudlets are found in the path (as expected in the BLR, for instance). However, unless the beam energy is very high this 


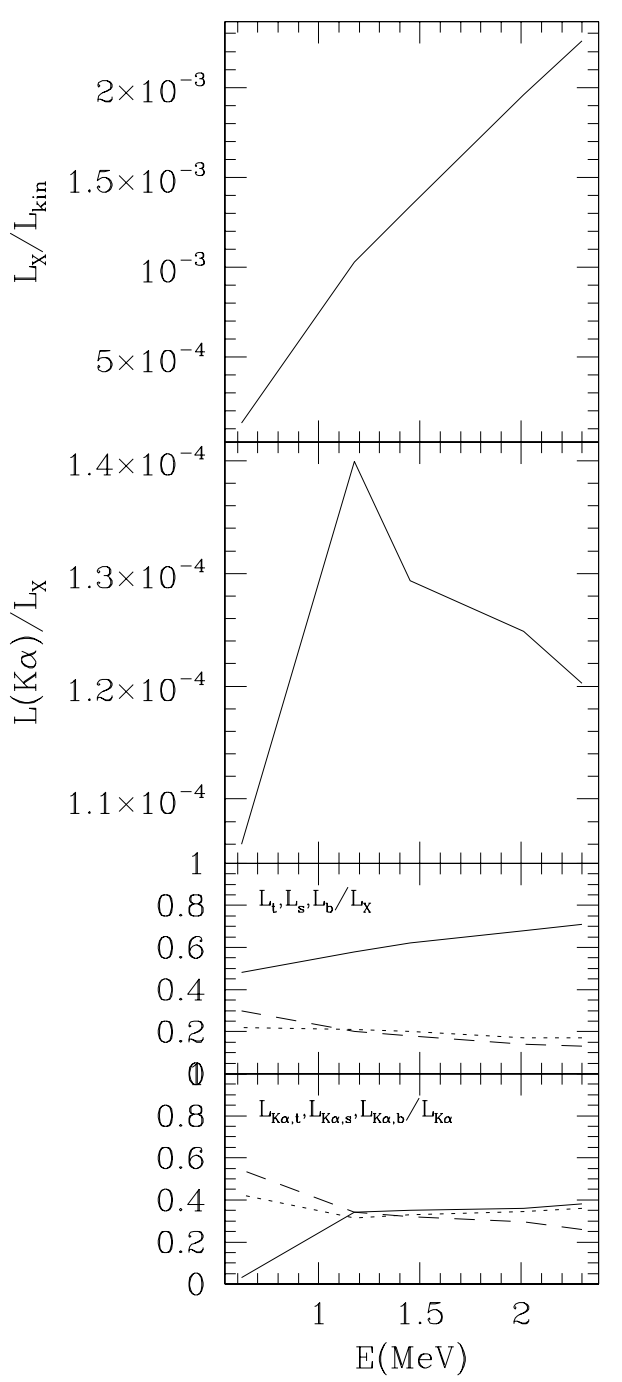

Fig. 5. Top: ratio between the total energy radiated in the $1-20 \mathrm{keV}$ range per electron and the kinetic energy of the electron plotted as a function of the mean kinetic energy of the impinging electrons. Middle: fraction of the X-ray (1-20 keV range) radiated in the $\mathrm{Fe} \mathrm{K} \alpha$ line. Bottom upper: fraction of the X-ray $(1-20 \mathrm{keV})$ continuum luminosity which is transmitted, scattered and reflected plotted with continuous, dashed and dotted lines, respectively. Bottom lower: as above but for the $\mathrm{Fe} \mathrm{K} \alpha$ line.

fraction is negligible; for instance only $\sim 1 \%$ of the incident electrons with energy $2.012 \mathrm{MeV}$ will reach the back of the $6 \times 10^{12} \mathrm{~cm}$ radius cloudlet and their average energy will have been degraded to $1.44 \mathrm{MeV}$.

Let us compare this value with the observations of a well-studied object such as MCG-6-30-15, a Seyfert I galaxy at $z=0.0078$. Recent observations with CHANDRA and XMM-Newton have allowed study of the $\mathrm{K} \alpha$ profile in detail resolving two clear components: a narrow component with $F_{\mathrm{K} \alpha}=2.5 \times 10^{-5}$ counts $\mathrm{s}^{-1} \mathrm{~cm}^{-2}$ and a broad component with $F_{\mathrm{K} \alpha}=2.0 \times 10^{-4}$ counts $\mathrm{s}^{-1} \mathrm{~cm}^{-2}$ (Lee et al. 2002; Fabian et al. 2002). From the expression above, the number of

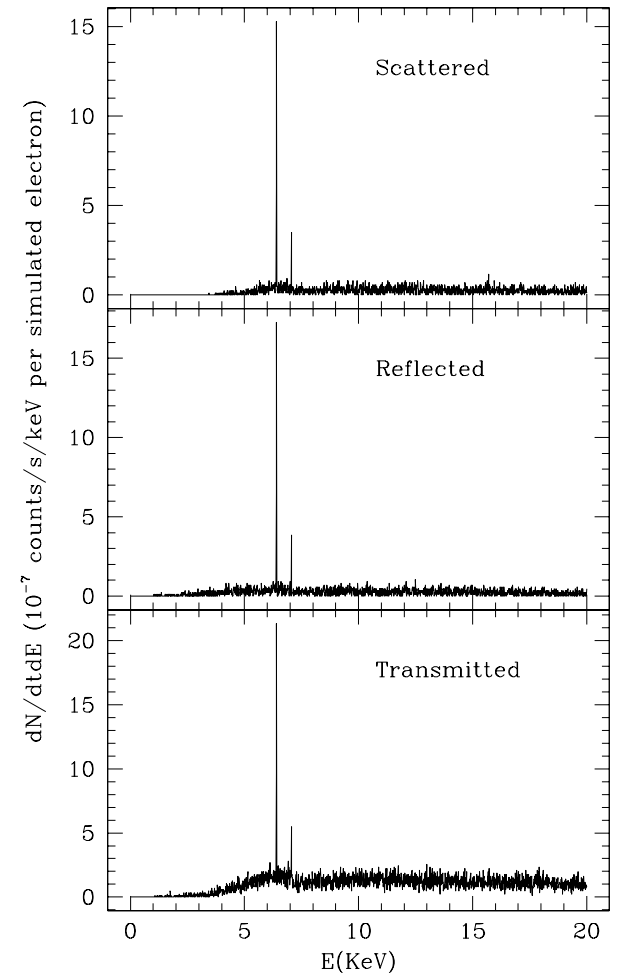

Fig. 6. Output X-ray radiation for an electron beam with energy $2012 \mathrm{keV}$, a beam radius of $1.23 \times 10^{5} \mathrm{~cm}$ impinging on a cloud with radius $6 \times 10^{12} \mathrm{~cm}$.

electrons with mean kinetic energy $\sim 1 \mathrm{MeV}$ required to produce the observed $\mathrm{Fe}-\mathrm{K} \alpha$ flux is:

$N_{\mathrm{e}}=9.62 \times 10^{55}\left(\frac{36 \mathrm{Mpc}}{1 \mathrm{Mpc}}\right)^{2}\left(\frac{F_{\mathrm{K} \alpha}}{\text { counts } / \mathrm{s} / \mathrm{cm}^{2}}\right)$

or, $N_{\mathrm{e}} \simeq 3.12 \times 10^{54}-2.49 \times 10^{55}$. This implies that the integrated kinetic luminosity of the beam should be:

$L_{\mathrm{KIN}}=1.6 \times 10^{-9}\left(\frac{\mathrm{erg}}{\mathrm{s}}\right) \frac{N_{\mathrm{e}} E(\mathrm{keV})}{\tau}$

where $\tau$ is the injection time (100 s, see Sect. 3). Henceforth, to reproduce the observed line flux the total kinetic luminosity of the beam should be, $L_{\mathrm{KIN}}=6.09 \times 10^{46}-4.68 \times 10^{47} \mathrm{erg} / \mathrm{s}$ or $\log L_{\mathrm{KIN}}=46.8-47.7$. Obviously, all this energy cannot be deposited in a single $6 \times 10^{12} \mathrm{~cm}$ cloud without evaporating it, but if the electron beams are distributed over the whole disk surface and the BLR they could provide a significant additional source of heating and line excitation.

Unfortunately, the electron kinetic luminosity can only be measured for radio-loud AGN (Celotti et al. 1997). If we use these values as a reference, $45<\log L_{\mathrm{KIN}}<48$, we found that they are comparable to the values derived above. Therefore, one can conclude that radio-loud AGNs are able to power electron beams to energies comparable to that required for electron collision to be a significant source of $\mathrm{Fe}-\mathrm{K} \alpha$ excitation.

The equivalent width of the $\mathrm{Fe}-\mathrm{K} \alpha$ line ranges from $0.1 \mathrm{keV}-0.4 \mathrm{keV}$ depending on the beam energy ${ }^{8}$ and the relative orientation to the observer; in general the equivalent width

${ }^{8}$ Only the simulations with beam energies $\geq 620 \mathrm{keV}$ are considered. 


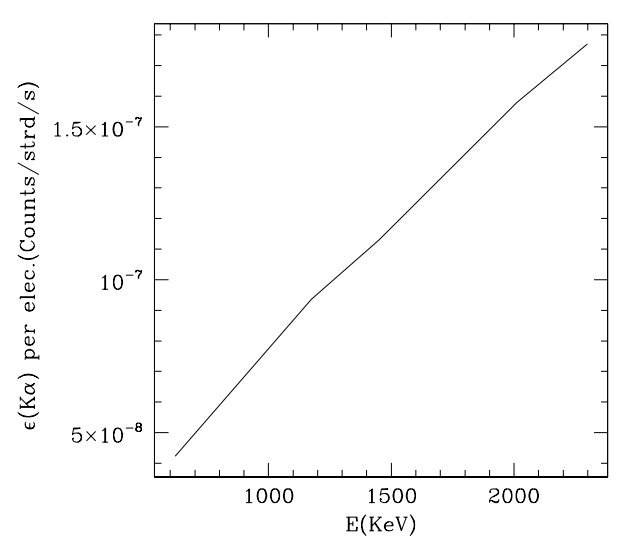

Fig. 7. Fe-K $\alpha$ yield $\left(\left\langle\epsilon_{\mathrm{K} \alpha}(E)\right\rangle\right)$ as a function of the kinetic energy of the electron beam.

is larger in the scattered than in the transmitted or reflected spectra. This range compares well with the observations that indicate $W\left(\mathrm{~K}_{\alpha}\right) \simeq 0.1 \mathrm{keV}$ in most of the AGNs (Guainazzi, private communication).

\subsection{The $X$-ray continuum}

The simulations (see Figs. 2-4 and 6) show that the relevance of electron collisions in the $1 \mathrm{keV}-20 \mathrm{keV}$ continuum spectrum is strongly dependent on the details of the model, especially on: (1) the relative orientation between the beam and the observer and (2) the radius of the electron beam compared to the characteristic radius of the absorbing medium. This affects not only the spectral energy distribution but also the strength of the continuum. Two important results come from Sect. 3:

At energies above the iron continuum bump the photon energy distribution is nearly flat. As a consequence, the photon in$\operatorname{dex}^{9}$ is expected to be $\Gamma \leq 2$ in the $8 \mathrm{keV}-20 \mathrm{keV}$ range (see Fig. 4). The contribution from reflected radiation could lower this number.

The ratio between continuum and line radiation depends on the ratio between the cloud $\left(R_{\mathrm{c}}\right)$ and the beam radius $\left(r_{\mathrm{b}}\right)$. If $r_{\mathrm{b}} / R_{\mathrm{c}} \ll 1$, the $\mathrm{X}$-ray continuum radiation is damped while the $\mathrm{Fe}-\mathrm{K} \alpha$ line emission is not significantly attenuated.

The precise determination of the output AGN spectrum is deferred to a further work since it requires a detailed modeling of the accretion disk atmosphere, the properties and location of the flares/lamppost and the properties of the BLR clouds. However some general characteristics may be derived from these simulations:

1. If the electrons are produced at some few tens $R_{\mathrm{S}}$ above the disk (e.g. the "lamppost" model), their interaction (inverse Compton scattering) with the strong radiation field from the accretion disk will brake them to non-relativistic speeds before reaching the disk. Relativistic electrons lose energy in the AGN environment by three main processes: inverse Compton scattering with the ambient radiation

\footnotetext{
${ }^{9} E F_{E} \propto E^{\Gamma}$ with $F_{E}=E \mathrm{~d} N / \mathrm{d} t / \mathrm{d} E$.
}

field, synchrotron radiation and Bremsstrahlung. Following Schlickeiser (2002), the energy loss rates are:

$$
\begin{aligned}
& \frac{\mathrm{d} E}{\mathrm{~d} t}_{\text {I.C. }}\left(\frac{\mathrm{erg}}{\mathrm{s}}\right)=2.33 \times 10^{-13} w\left(\mathrm{erg} / \mathrm{cm}^{3}\right) \\
& \frac{\mathrm{d} E}{\mathrm{~d} t}{ }_{\text {Sync }}\left(\frac{\mathrm{erg}}{\mathrm{s}}\right)=3.49 \times 10^{-13} \frac{B_{\perp}^{2}(\mathrm{G})}{8 \pi} \\
& \frac{\mathrm{d} E}{\mathrm{~d} t}_{\text {Brems }}\left(\frac{\mathrm{erg}}{\mathrm{s}}\right)=5.35 \times 10^{-11} \frac{n_{\mathrm{H}}\left(\mathrm{cm}^{-3}\right)}{10^{11} \mathrm{~cm}^{-3}}
\end{aligned}
$$

respectively, with $w$ the energy density of the radiation field, $B$ the magnetic field and $n_{\mathrm{H}}$ the density. The energy density is roughly,

$w=\frac{L}{c r^{2}}=9.58 \times 10^{3} \frac{\mathrm{erg}}{\mathrm{cm}^{3}} \frac{L}{10^{44} \mathrm{erg} / \mathrm{s}}\left(\frac{r}{20 R_{\mathrm{S}}}\right)^{-2}$

and as a result,

$$
\frac{\mathrm{d} E}{\mathrm{~d} t} \text { I.C. }\left(\frac{\mathrm{erg}}{\mathrm{s}}\right)=2.23 \times 10^{-9}\left(\frac{r}{20 R_{\mathrm{S}}}\right)^{-2}
$$

(significantly larger than the Bremsstrahlung loss rate assuming that the environment is at least as diffuse as the BLR clouds). As the energy of the electrons is $\sim 1 \mathrm{MeV}$; this indicates that the electron becomes non-relativistic in a time scale of $\sim 700 \mathrm{~s}$, e.g. before reaching the disk. In the process, the radiation from the disk becomes more energetic since after each inverse Compton scattering (in this Thomson limit) its energy increases by an average factor of $\sim(4 / 3) \gamma^{2}$. As a result a far UV bump is generated in the energy distribution. An electron flux of $\sim 10^{54}$ particles per second (see above) produces a UV bump with a peak flux of $5.0 \times 10^{45} \mathrm{erg} / \mathrm{s} / \mathrm{keV}$ at a few hundreds Angstroms.

Thus, relativistic electrons could not produce significant $\mathrm{X}$-ray emission by impact with the disk unless the electrons are accelerated in the path from the "lamppost". So, we might expect that the dominant X-ray contribution comes from the interaction with the BLR cloudlets which are at a characteristic distance of $10^{16} \mathrm{~cm}\left(\simeq 3.4 \times 10^{2} R_{\mathrm{S}}\right)$. Even in this case, braking by inverse Compton scattering is expected to be important close to the "lamppost" because of the strong radiation field. Notice that if hard X-ray radiation is produced at the "lamppost" it will be made even harder by the interaction with the electrons (for instance $10 \mathrm{keV}$ photons will be scattered to $11.7 \times 10 \mathrm{keV}$ ). A good understanding of the precise physical processes in the "lamppost", including magnetic fields and density-opacity effects, is required to properly evaluate the number of relativistic electrons that may arrive at the BLR.

2 . If the electrons are produced in flares at roughly one scaleheight $\left(\sim 10^{11} \mathrm{~cm}\right)$ above the disk, the most important contribution to the X-ray spectrum comes from the disk; it will be associated with the Bremsstrahlung radiation ("reflected spectrum") produced at the location where the beam impinges on the disk. In this case, the strength of the X-ray emission depends on the relative orientation between the disk and the line of sight. 
In both cases, the electron energy is damped into the heating of the dense gas. As a result, hot spots are produced. The analysis of the role of this energy in the BLR heating (and in the cloud evaporation) as well as in the disk stability has to be studied. Therefore, a detailed modeling of the AGN is required to determine properly the expected output spectrum and its energy.

\section{Discussion: How do particles fit in the current "radiation paradigm"?}

It is widely accepted that photons are the primary output from the release of gravitational energy in the accretion process in AGNs. As a result, only "photon-matter" interaction has been considered to interpret the spectrum of AGNs; this represents the so-called "radiation paradigm". The contribution of particle acceleration to the release of energy is neglected except in the case of observed jets; as an example the weak radio emission from radio-quiet AGNs is thought to represent compact (or unresolved) jets (Anderson et al. 2004).

This assumption ignores that accretion disks may not be purely hydrodynamical entities and that magnetic fields may play a significant role in angular momentum transport. However observations (the magnetized radio jets), suggest that fields may be relevant. In this case energy dissipation implies the generation of high energy photons and particles (the Sun is a good example of that). The onset of the magneto-rotational instability in the accretion disk acts as a dynamo and field buoyancy leads to the formation of a magnetized corona. The interaction of the rising loops with the ambient magnetic field naturally produces flares, e.g. high energy photons (see Różańska et al. 2004, for a recent analysis of their contribution to the $\mathrm{X}$-ray spectrum) and electrons beams. However, no obvious structure in the coronal field is expected and it is still unclear whether a large-scale, ordered (ambient) field is required to channel the outflow into a collimated jet (see Hawley 2004).

In this work, we have shown that the "reflected" disk radiation, after heating by high energy electron impact, might be significant; this also applies to the scattered radiation from the impact with the BLR clouds. However, two key questions remain open:

1. What is the average energy of the electrons (or in turn, what is the detailed physics behind the flaring process)?

2 . Is it reasonable to assume that $10^{54-55}$ electrons with kinetic luminosities comparable to radio jets are produced in coronal flares in disk? Furthermore, why do they become collimated into single, large-scale jets?

Answering the first question is beyond the scope of this work but some hints to answer the second question could come from the research in another type of accreting source: protostars. Accretion disks are always observed around protostars but jets seem to be detected only when the disk axis is roughly parallel to the ambient magnetic field (Ménard \& Duchêne 2004). If this general physics also applies to AGNs we might naively expect that the initial electron kinetic luminosity is similar in radio-loud and radio-quiet AGNs. The main difference would be that in radio-loud AGNs the electrons are channeled (and accelerated) in a coherent large scale outflow. As a result, the
$\mathrm{X}$-ray luminosity should be higher in radio-quiet than in radioloud AGNs because in the first case most of the electrons would release their energy close to the AGN by collisions with the environment while in radio-loud AGNs most of the energy would be released in the large-scale outflow ${ }^{10}$. This would provide a simple explanation of why the "X-ray reflection features" are weaker in radio-loud than in radio quiet AGNs (Ballantyne et al. 2002).

Moreover, the X-ray fluctuations appear to be random but not completely scale-invariant. In MCG-6-30-15 time lags ( $\leq 200 \mathrm{~s}$ ) are detected between energy bands, with soft variations leading the hard (Vaughan et al. 2003). This is the standard behavior in stellar flares; magnetic reconnection is produced in several steps; first, the gas in the current layer is heated by the shock energy released at the layer borders, after that the onset of turbulence produces strong electric fields which accelerate particles to relativistic speeds, generating X-ray radiation when these particles shock with the stellar surface. The radiation released in the first step is softer than in the second, because thermal heating produces a rather complete redistribution of the energy among all the intervening particles.

Unfortunately, the strong radiation field around the central black hole may blur this trend in radio-loud AGNs since inverse Compton scattering of the relativistic electrons in the jet also produces strong X-ray radiation in the range considered. Jet electrons are highly relativistic with $\gamma \gg 1$, thus, the differential cross-section for Compton scattering is given by the KleinNishina formula. In this extreme regime the scattered photons carry away a large fraction of the electron energy.

There is however, a feasible test of this hypothesis. Photoionization (photoelectric cross-sections) for the $\mathrm{K}$ shell electrons depends on the atomic number as $Z^{5}$ (Sauter 1931; Heitler 1954) while high energy photon emission by the bremsstrahlung of a relativistic electron depends on $Z^{2}$. Therefore, if several K-shell lines of different species are detected in a given $\mathrm{X}$-ray spectrum, the lines ratios could be used to find out the relevance of particles in gas ionization (Gómez de Castro \& Antonicci, in preparation).

\section{Conclusions}

The question addressed in this work is whether some of the features observed in the X-ray spectrum of AGN could be produced by the interaction of highly relativistic particles with the circumnuclear gas. We show that, even for particles with rather low energies $(\leq 2.2 \mathrm{MeV})$, the Fe-K $\alpha$ flux and the $\mathrm{X}$ ray continuum may be significantly affected by the energy cascade induced by particle showers. The net effect is to produce a "hot X-ray continuum" and excess Fe-K $\alpha$ flux compared to models that only take into account photoionization. The two physical parameters controlling this process are the kinetic energy of the electrons and the spatial distribution and properties of the absorbing medium. This work is a first step towards a

\footnotetext{
${ }^{10}$ Notice again the analogy with solar flares: X-ray radiation from the electrons colliding with the solar surface is always detected; radioemission depends on whether the electrons get trapped in an open field line leaving the flare region.
} 
detailed modeling of the interaction between the circum-blackhole environment with the high energy particles produced by the AGNs.

The remaining key question is: what is the particle flux (and its energy distribution) generated in an accreting black hole? The analysis of $\gamma$ ray radiation from AGNs will provide us with important clues.

Acknowledgements. We thank Willem Wamsteker, Matteo Guainazzi, J. J. Honrubia and Luis Conde for useful discussions. This work has been partially financed by the Ministry of Science and Technology of Spain through grant AYA2000-966.

\section{References}

Anderson, J. M., Ulvestad, J. S., \& Ho, L. C. 2004, ApJ, 603, 42

Antonicci, A. 2004, Ph.D. Thesis, Universidad Complutense de Madrid

Balbus, S. A., \& Hawley, J. F. 1991, ApJ, 376, 214

Ballantyne, D. R., Ross, R. R., \& Fabian, A. C. 2002, MNRAS, 336, 867

Ballantyne, D. R., \& Fabian, A. C. 2003, ApJ, 592, 1089

Baró, J., Sempau, J., Fernandez-Varea, J. M., \& Salvat, F. 1994, Nucl. Instr. Meth. B, 84, 465

Bethe, H. A., \& Heitler, W. 1934, Proc. Roy. Soc. London A, 146, 83
Celotti, A., Padovani, P., \& Ghisellini, G. 1997, MNRAS, 286, 415

Collin, S., Coupe, S., Dumont, A.-M., Petrucci, P.-O., \& Rozanska, A. 2003, A\&A, 400, 437

Czerny, B., Różańska, A., Dovciak, M., Karas, V., \& Dumont, A.-M. 2004, A\&A, 420, 1

Fabian, A. C., Vaughan, S., Nandra, K., et al. 2002, MNRAS, 335, L1

Hawley, S. 2004, Ap\&SS, 291

Heitler, W. 1954, The quantum theory of radiation (Oxford: Oxford Univ. Press)

Heyvearts, J., Priest, E. R., \& Rust, D. M. 1977, ApJ, 216, 123

Hombourger, C. 1998, J. Phys. B: At. Mol. Opt. Phys., 31, 3693

Lee, J. C., Iwasawa, K., Houck, J. C., et al. 2002, ApJ, 570, L47

Menard, F., \& Duchene, G. 2004, Ap\&SS, 291

Petrucci, P. O., Henri, G., Maraschi, L., et al. 2003, A\&A, 388, L5

Pounds, K. A., Nandra, K., Stewart, G. C., George, I. M., \& Fabian, A. C. 1990 , Nature, 344, 132

Pelletier, G., \& Pudritz, R. E. 1992, ApJ, 394, 117

Peterson, B. M. 1997, An introduction to active galactic nucleii (Cambridge Univ. Press), 37

Sauter, F. 1931, Ann. Phys., 9, 217

Schlickeiser, R. 2002, Cosmic Ray Astrophysics (Berlin: SpringerVerlag)

Sung-Joon, Ye, Brezovich, I., Pareek, P., \& Naqvi, S. A., Phys. Med. Biol., 49, 387

Vaughan, S., Fabian, A. C., \& Nandra, K. 2003, MNRAS, 339, 1237

Verner, D. A., \& Yakovlev, D. G. 1995, A\&AS, 109, 125 